\title{
0 Acompanhamento Terapêutico (AT): dispositivo de atenção psicossocial em saúde mental ${ }^{*}$
}

Ana Celeste de Araújo Pitiá

Antonia Regina Ferreira Furegato

PITIÁ, A.C.A.; FUREGATO, A.R.F. Therapeutic Accompaniment (TA): tool for psychosocial care within mental healthcare. Interface - Comunic., Saude, Educ., v.13, n.30, p.67-77, jul./set. 2009.

This paper discusses the clinical practice of Therapeutic Accompaniment (TA), incorporated into the psychosocial care model within mental healthcare and into other such models. The aim was to discuss the process of psychosocial rehabilitation and this type of therapeutic action within mental healthcare, in relation to TA clinics. For this, the paradigms of the asylum and psychosocial care models that are the reference points for intervention practices in mental healthcare were examined from a theoretical viewpoint. These reflections showed the importance of consolidating a care network that makes it possible for patients with mental suffering to have prospects within their lives. This is a challenge to be faced, and the priority should be the subjects involved in this and their context of social immersion.

Keywords: Therapeutic accompaniment. Psychosocial rehabilitation. Psychiatric nursing. Mental healthcare.
Este artigo aborda a prática clínica do Acompanhamento Terapêutico (AT), incorporado ao modo de atenção psicossocial em saúde mental e seus outros dispositivos. Objetiva-se discutir o processo de reabilitação psicossocial e este tipo de ação terapêutica na saúde mental, com base na clínica de AT. Para tanto, realizouse uma reflexão teórica, considerandose os paradigmas dos modos asilar e psicossocial de atenção, que referenciam as práticas de intervenção terapêutica em saúde mental. As reflexões conduzem à importância da consolidação de uma rede de atenção que possibilite perspectiva de vida aos portadores de sofrimento psíquico como um desafio a ser enfrentado que considere prioritariamente o sujeito aí implicado e seu contexto de imersão social.

Palavras-chave: Acompanhamento terapêutico. Reabilitação psicossocial. Enfermagem psiquiátrica. Saúde mental.

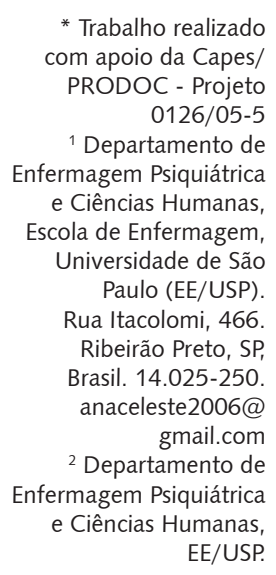

* Trabalho realizado com apoio da Capes/ PRODOC - Projeto 0126/05-5 ${ }^{1}$ Departamento de Enfermagem Psiquiátrica e Ciências Humanas, Escola de Enfermagem, Universidade de São Paulo (EE/USP). Rua Itacolomi, 466 Ribeirão Preto, SP, Brasil. 14.025-250 anaceleste2006@ gmail.com 2 Departamento de Enfermagem Psiquiátrica e Ciências Humanas, $\mathrm{EE} / \mathrm{USP}$. 


\section{Introdução}

O impacto do transtorno mental, avaliado, notadamente, pela sobrecarga sobre o indivíduo, a família e a sociedade, indica a relevância do tema para a agenda internacional (World Health Organization, 2002).

A Organização Mundial da Saúde divulgou, em um relatório, que até países desenvolvidos se esquecem de cuidar da mente humana. Ressalta que, apesar dos transtornos mentais atingirem cerca de 450 milhões de pessoas, ainda estão longe de receber a mesma relevância dada à saúde física, sobretudo nos países em desenvolvimento. Comparando-se os transtornos mentais com as principais doenças que afetam a população, estes respondem por $12 \%$ das condições de saúde que sobrecarregam os países pobres e médios em riqueza Os serviços e as estratégias de promoção e proteção da saúde mental precisam ser coordenados entre si e articulados com outros serviços, tais como: a segurança social, a educação, o emprego e a habitação. As ações para a saúde mental devem ser monitoradas e analisadas para que as decisões possam ser constantemente ajustadas para responderem aos desafios que se apresentam (Organización Panamericana de la Salud, 2003).

No Brasil, o Estado tem assumido papel decisivo na reestruturação da atenção psiquiátrica, desde 1987, com a 1a.Conferência Nacional de Saúde, recebendo reforços com a Declaração de Caracas, na Venezuela de 1990. Desde então, os países latino-americanos vêm envidando esforços para a promoção de serviços comunitários - substituindo o manicômio -, integrados à rede de serviços de saúde em atenção primária à saúde e suas redes sociais, priorizando a manutenção do doente em seu meio social (Brasil, 2004).

A principal repercussão desta proposta é que a rede básica de saúde passa a ser o principal meio para o atendimento de portadores de transtornos mentais que adoecem ou que estão em algum tipo de acompanhamento, e o profissional da saúde passa a se ocupar de um contexto ampliado onde ocorre o cuidado.

A assistência em saúde mental propõe a atenção descentralizada, interdisciplinar e intersetorial, bem como vincula o conceito de saúde mental aos conceitos de cidadania e produção de vida, gerando transformações nas concepções e práticas de saúde mental, na organização dos serviços, na formação e na capacitação dos profissionais da área. No sentido instrumental, o processo de reabilitação psicossocial representa um conjunto de meios (programas e serviços) que se desenvolvem para facilitar a vida das pessoas com problemas mentais severos e persistentes. Esse processo destina-se a restaurar o melhor nível possível de autonomia do indivíduo, no exercício de suas funções sociais (Pitta, 2001).

As práticas de atenção psicossocial são tributárias de diferentes movimentos sociais e científicos em vários campos teóricos, articulados de forma complexa e exigindo incursões pelas teorias da análise política de instituições, da análise institucional e da constituição subjetiva. Incluem elementos de análise da história dos principais movimentos institucionais internacionais no campo da saúde mental e suas repercussões no contexto brasileiro: psiquiatria de setor e psicoterapia institucional na França; antipsiquiatria e comunidades terapêuticas na Inglaterra; saúde mental comunitária nos EUA, e o movimento democrático da desinstitucionalização na Itália (Costa-Rosa, 2000).

Deixando de se ocupar apenas com a doença, com a prescrição de medicamentos e aplicação de terapias, os profissionais da atenção psicossocial passam a se ocupar dos sujeitos que precisam de tratamento e com a qualidade do cuidado oferecido. Passam a ocupar-se também do cotidiano, do tempo, do espaço, do trabalho, do lazer, do ócio, do prazer e da organização de atividades conjuntas. Em consonância com o processo de reabilitação, espera-se que os profissionais trabalhem enfatizando as partes mais sadias e as potencialidades do indivíduo, mediante uma abordagem compreensiva, oferecendo suportes: vocacionais, sociais, recreacionais, residenciais, educacionais, ajustados às demandas singulares de cada indivíduo (Pitta, 2001; Furegato, 2000; Amarante, 1999). 
${ }^{3}$ Utiliza-se, como convenção, a denominação AT (Acompanhamento Terapêutico) para a prática clínica, e at (acompanhante terapêutico) quando se tratar do profissional que exerce a prática.
O objetivo deste artigo é apresentar uma reflexão sobre a clínica AT, baseada na experiência das autoras com a clínica do Acompanhamento Terapêutico (AT) ${ }^{3}$ e na literatura sobre o tema, bem como levantamentos bibliográficos sobre as políticas, papéis e funções dos serviços de saúde mental, por intermédio do SIBI Sistema de Bibliotecas da USP, do site do Ministério da Saúde e OPAS, da BIREME e da biblioteca da USP - Ribeirão Preto.

Foi considerado, como pano de fundo para esta reflexão, o questionamento sobre o modo asilar em contraste com o modo psicossocial, contexto contemporâneo de reformulações dos paradigmas da atenção em saúde mental. Dessa maneira, discute-se a clínica do Acompanhamento Terapêutico (AT) como um dos dispositivos de ação terapêutica que se incorpora à atenção em saúde mental, procurando promover a reintegração psicossocial dos portadores de sofrimento psíquico, tendo por base a experiência vivenciada em Ribeirão Preto, que gerou uma tese de doutorado (Pitiá, 2002) e cursos de formação em AT na Escola de Enfermagem da USP.

Para contextualização, tomam-se, como referência, os dispositivos do modo psicossocial, a exemplo dos CAPS (Centros de Atenção Psicossocial), convergentes com a idéia de se configurarem em modos estratégicos para reversão do modelo centrado no hospital, conforme descrito por OnockoCampos e Furtado (2006).

\section{O modo asilar e o modo psicossocial de atenção em saúde mental}

O saber sobre a loucura, até o final do século XIX, era, em essência, baseado no conhecimento sobre a doença, e não sobre o homem doente. O guia do processo terapêutico era o quadro sintomatológico e a intervenção era marcada pela alienação social do sujeito referenciada para o contexto hospitalar. Nesse período, o homem era apenas um terreno onde se instalava a doença, onde evoluíam os sintomas e sobre o qual o poder de controle era exercido.

No processo histórico das mudanças de paradigmas das práticas em saúde mental e, sob as influências da Sociologia, da Antropologia, da Filosofia e da Psicanálise, a abordagem sobre a loucura mudou de foco - passando da doença para o doente - sendo, então, o homem visto em sua fala e corporificando a doença.

Dessa forma, o contexto da Reforma Psiquiátrica apresenta um conjunto de medidas que objetivam desospitalizar o atendimento em saúde mental e a desconstrução do modo asilar de atendimento, provocando a transformação da concepção do hospital psiquiátrico como única forma de cuidado. Assim, a exclusividade das decisões e das ações terapêuticas deixa de se concentrar nas mãos do médico, passando a ser da responsabilidade de todos os profissionais (médicos, enfermeiros, assistentes sociais, terapeutas ocupacionais, psicólogos), como também de grupos da comunidade não-psiquiátricos, tais como os grupos de autoajuda e os religiosos e clérigos (Amarante, 1999; Lobosque, 1997).

Os princípios da desconstrução assistencial e cultural do manicômio vêm provocando o rompimento do paradigma hospitalar de atenção em saúde mental, marcado por momentos de maior e menor intensidade de ações transformadoras, tal como ocorre em todos os processos de mudança.

O resultado da reorientação do modelo assistencial em saúde mental no país implica reorganização da estrutura dos serviços, composição das equipes de saúde de nível superior e médio, bem como de portadores de transtornos mentais e seus familiares, usuários dos serviços de saúde mental. Estas mudanças estão 
marcadas pela responsabilização do Ministério da Saúde neste movimento, por meio de leis, portarias e regulamentações (Aranha Silva, Fonseca, 2005; Brasil, 2004).

Os conceitos sobre as abordagens terapêuticas relativas à doença mental e ao sofrimento psíquico vão sendo recriados no processo de subjetivação destes tratamentos. Pela concepção que vem sendo construída sobre os atendimentos, o sujeito doente tem a possibilidade de ser avaliado e atendido por meio de um modo de atenção mais singular e ético. O foco passa a ser a saúde mental e, nesse sentido, a escuta passa a ser valorizada, sustentando-se a possibilidade de se acolherem diferentes formas de subjetivação, representadas pelas maneiras singulares de se estar no mundo (Bronz et al., 1997).

Este processo de transformação é lento e irregular, pois, apesar da criação dos serviços, da regulamentação das equipes e da normatização das ações, há a necessidade de serem desconstruídas concepções manicomiais, instaladas nas mentalidades dos próprios profissionais, o que influencia, em grande medida, a qualidade do atendimento prestado aos usuários.

Como formas alternativas ao manicômio e à internação total, são implementados espaços de atendimento como: ambulatórios, centros de atenção (CAPS), hospitais-dia, unidades psiquiátricas de internação e de emergências em hospitais gerais, além de outros recursos - como oficinas terapêuticas, grupos de convivência, lares abrigados, cooperativas de trabalho e a prática clínica do AT. Estes recursos vão ao encontro do processo de subjetivação e de transformação não apenas da assistência, mas da própria concepção de transtorno mental e de sofrimento psíquico.

No contexto atual das transformações da assistência em saúde mental, é importante uma reflexão sobre os dois modos das práticas de atenção que se opõem: o modo asilar e o modo psicossocial (Costa-Rosa, 2000). Estes modelos têm características peculiares que contextualizam historicamente as práticas de tratamento em saúde mental, onde se pode destacar o AT.

O modo asilar é caracterizado pela presença do manicômio, tecnicamente sustentado pela contenção química abusiva que favorece o ambiente de clausura do "louco", um personagem social discriminado, excluído e recluso, limitado à loucura e à desrazão. Na lógica manicomial, os hospitais psiquiátricos tendem a uma política assistencial que se baseia em princípios de verdade exclusiva, de incurabilidade e de segregação (Sampaio, Santos, 2001).

Essa concepção de doente mental tem sua origem em meados do século XVII, no mesmo processo em que foram excluídos todos os que subvertiam a ordem pública - como os pobres, velhos, inválidos, mendigos que transgrediam as leis -, ou seja, enquadravam-se no segmento da população a quem se podia aplicar castigos públicos, destinados a "corrigir" a ociosidade e a desrazão, que desorganizava a ordem vigente (Amarante, 1995; Foucault, 1993).

$\mathrm{Na}$ literatura sobre a história clássica da loucura, o hospital se constituiu no instrumento terapêutico pautado em saberes e discursos que fundaram a psiquiatria, a partir do final do século XVII. Foi inventado por meio de dispositivos disciplinares da prática médica, com a classificação e sistematização das doenças, o que levou ao desenvolvimento do atendimento psiquiátrico clássico, resumido em internação em ambientes "apropriados" aos loucos e suas manifestações psíquicas. Essa proposta encerra um poder institucional com o mandato social da assistência e tutela do louco. A instituição trouxe consigo as funções de controle/exclusão social, ordem institucional, racionalização e objetivação de recursos (Aranha Silva, Fonseca, 2005; Foucault, 1993).

Em suma, o modelo asilar tem sido tradicionalmente custodial, médico-centrado, subsidiado pela ação dos demais profissionais, especialmente da enfermagem (Stefanelli, 2008); conformando uma organização técnica do trabalho que divide a atenção coletiva, com a finalidade de "cura", considerando apenas a adaptabilidade social do sujeito portador do transtorno mental, tornando-o objeto de intervenção, e deixando, nas mãos do médico, a posse exclusiva da condução das medidas terapêuticas para essas pessoas (Aranha Silva, Fonseca, 2005).

Assim, no duro regime asilar, os hospitais psiquiátricos caracterizam-se pelo aspecto de grandes estruturas físicas e fechadas, com capacidade crescente para abrigarem todos os sujeitos que se enquadram nos princípios do asilamento/confinamento. O hospital psiquiátrico tornou-se uma instituição onde se estabelecem relações de dominação/submissão, gerando sofrimento a todos e, em especial, ao enfermo (Pitiá, 1997). Por estes motivos, os últimos cinquenta anos têm sido palco de intensas transformações na concepção da saúde e da doença mental, bem como de seus tratamentos. 
Na política dessas transformações, muitas mudanças vêm ocorrendo, e "desinstitucionalizar" emergiu como um conceito que tem norteado o processo da Reforma Psiquiátrica. Baseando-se nos pontos principais da Declaração de Caracas, Alves (2001) sinaliza para a diversificação das possibilidades de oferta de serviços, superando a crença do hospital psiquiátrico como ideal para tratamento e única opção. O hospital deve oferecer tratamento de fato, e não confinamento, tendo, assim, maneiras de se respeitarem e ampliarem os direitos das pessoas com transtornos mentais, incorporando-as como sujeitos ao processo de superação de suas dificuldades. Ao mesmo tempo, pressupõe-se a desconstrução do arcabouço teórico da psiquiatria clássica, introduzindo novas formas de lidar com o indivíduo portador de transtorno mental. Abre-se espaço para outro paradigma de atendimento, que considera o sofrimento psíquico e a rede de relações da pessoa, pensando o seu espaço social como contexto das subjetividades.

O modo psicossocial passa a ser o novo paradigma de atendimento em saúde mental. Nele, o processo de reabilitação constitui uma estratégia que implica algo mais do que, simplesmente, passar um usuário/paciente de um estado de "desabilidade" a um estado de "habilidade" e/ou de um estado de incapacidade para um estado de capacidade. A reabilitação psicossocial é uma estratégia global, múltipla, ética e de solidariedade, que ajuda os sujeitos a lidarem com sua doença em meio aos seus afazeres cotidianos, sua contratualidade afetiva, social, econômica, e viabiliza o melhor nível possível de autonomia para a vida em comunidade (Pitta, 2001; Saraceno, 1998).

O modo psicossocial de atenção pode ser conceituado com base em quatro parâmetros: 1) pelas formas de divisão do trabalho interprofissional que preconizam a implicação subjetiva do usuário como condição básica para maior aproximação nas relações dos profissionais com os usuários e a população da área atendida; 2) pelas formas de organização das relações intrainstitucionais, convergentes com as relações interprofissionais, preservando-se o poder decisório de origem políticooperacional, quando há que se estimular a comunicação entre profissionais dos serviços no teor de deliberações das ações executáveis na rede de atendimento; 3 ) pelas modalidades de relacionamento com os usuários e a população, preconizando a integralidade das ações no território; 4) pelas implicações éticas dos efeitos das práticas em saúde mental e quanto consideram o desejo do sujeito e a dimensão dos ideais pretendidos pela pessoa do usuário, considerando-se seus projetos de vida (Costa-Rosa, Luzio, Yasui, 2001; Costa-Rosa, 2000).

As práticas do modo psicossocial são decorrentes de diferentes movimentos sociais e científicos e de vários campos teóricos que fizeram eco às propostas de transformação do modelo (Costa-Rosa, 2000; Amarante, 1999; Saraceno, 1999). É no contexto do modo psicossocial de atenção à saúde que se insere a prática clínica do AT, como um instrumento de ação terapêutica que considera o portador de transtornos mentais em seu contexto sócio-histórico-psíquico-biológico e cultural, com vistas a contribuir com o processo de inclusão das diferenças, do bizarro, das dificuldades humanas.

\section{Dispositivos de ação terapêutica na saúde mental}

Atualmente, o modelo hospitalocêntrico é ainda hegemônico no Brasil e o grande desafio é reorientar esse modelo de atenção para além dos hospitais psiquiátricos, desconstruindo saberes arraigados há vários séculos. Como já foi dito, a perspectiva é a criação de novas culturas de convivência com o doente mental, devolvendo-lhe o direito à vida, à liberdade e à cidadania (CostaRosa, Luzio, Yasui, 2001). Dessa forma, o atendimento hospitalar passa a ter uma função restrita aos aspectos impactantes da crise dos portadores de sofrimento psíquico, possibilitando seu rápido acesso ao próprio contexto social. Isto impõe eficiência terapêutica e agilidade nos procedimentos técnicos.

Representando uma forma de assistência que nasce para contrapor o modelo hospitalocêntrico, o CAPS (Centro de Atenção Psicossocial) se configura como espaço de atendimentos realizados por uma equipe multiprofissional, destinada a atender os problemas de saúde mental, individuais e coletivos (Vianna, Barros, 2005). Distingue-se, especialmente, pela horizontalidade de ações interdisciplinares e terapêuticas, que favorecem a inclusão do sujeito portador de um transtorno mental em seu contexto social, aliviando o seu sofrimento. 
O CAPS é parte do Sistema Único de Saúde (Brasil, 2004). Sampaio e Santos (2001) descrevem suas características como: a) integração de sistemas primários e secundários de atenção; b) acessibilidade local (distrital ou municipal) e integração a sistemas de política social com interação direta e cotidiana com as famílias; c) prática multiprofissional e articulação de saberes da Enfermagem, Medicina, Psicologia, do Serviço Social e da Terapia Ocupacional, voltados ao atendimento e solução dos problemas pessoais, grupais e sociais, determinantes e determinados por alterações de comportamento e da afetividade; d) multiplicidade de práticas e críticas às práticas por meio de pesquisas sistemáticas, sempre reinventando, sem dogmatismos, sem uma teoria única de tratamento; e) processo de supervisão sistemática, institucional e técnica da organização, dos programas e das integrações profissionais; f) centro dinâmico das políticas de saúde mental, revolucionando a atitude dos profissionais de saúde mental e da sociedade frente às concepções de doença mental e dos direitos de cidadania.

Nos municípios de maior porte, além dos CAPS, para atendimentos de adultos, e CAPS infantis, a rede de serviços públicos também conta com ambulatórios de saúde mental, cuja principal função é a assistência aos usuários, portadores de transtornos mentais, e seus familiares, incluindo: análise, diagnóstico, tratamento, cuidado e reabilitação, objetivando sua reinserção na comunidade. Atende todas as patologias psiquiátricas e do comportamento, com exceção dos portadores de farmacodependências, os quais são atendidos em serviços denominados de CAPS-AD (Brasil, 2004).

Os Ambulatórios de Saúde Mental oferecem atendimento médico, de enfermagem e psicológico, bem como visita domiciliar aos portadores e grupos de familiares, coordenados pela enfermeira e pela assistente social, a exemplo do Ambulatório Regional de Saúde Mental do município de Ribeirão Preto (Osinaga, Furegato, Santos, 2005). Em municípios onde não há ambulatórios psiquiátricos, tais intervenções terapêuticas são realizadas nos CAPSs.

Outras unidades de atendimento em Saúde Mental conformam-se em estruturas institucionais de semi-internação durante o dia, denominadas de Hospitais-dia, e descritas como locais que oferecem, ao portador de transtorno mental, a possibilidade de manter vínculos familiares e com a comunidade que o cerca. As atividades focalizam: os atendimentos clínicos, cuidados de enfermagem, atendimentos psicológicos, grupos terapêuticos, oficinas terapêuticas, esportes, terapia corporal, trabalho nas redes sociais, atividades comunitárias (passeios externos, atividades culturais e artísticas, festas e celebrações de datas comemorativas), grupos de chegada e saída, além das reuniões e atendimentos que incluem os familiares.

$\mathrm{Na}$ atenção básica, pelas políticas públicas atuais, procura-se fomentar experiências de atendimento em saúde mental, nos serviços das Unidades Básicas de Saúde (UBS) e pela Estratégia de Saúde da Família (ESF). As equipes ocupam os vários espaços extrainstitucionais, de acompanhamento dos tratamentos do portador de transtorno mental, detecção e encaminhamento de casos suspeitos, indo ao encontro da família e suas dificuldades, procurando incluir todos os problemas relativos ao sofrimento psíquico. Quando a ESF se responsabiliza pelos portadores de sofrimento psíquico severo, podem ser observados resultados como: a diminuição no número de reinternações psiquiátricas das pessoas acompanhadas, promoção da saúde mental e reintegração para pacientes psicóticos. Nas intervenções domiciliares nos períodos de crise, o profissional que atende a família dá ênfase à capacidade de escuta, resolução de problemas e favorecimento da reinserção psicossocial (Souza, 2004), fazendo, muitas vezes, a função de terapeuta de AT.

Essa maneira de se prestar o atendimento na ESF tem compatibilidades com a prática clínica no AT, no que diz respeito, tanto à predominância do trabalho extrainstitucional, estando ao lado do sujeito e suas dificuldades, dentro do seu contexto de vida, bem como pela ênfase na capacidade de escuta, promovendo-se o acolhimento às próprias possibilidades de saídas para o sofrimento, que tenham significado para o sujeito.

\section{A ação terapêutica do Acompanhamento Terapêutico (AT)}

A ação terapêutica nos dispositivos extra-hospitalares, por meio da estratégia global do processo de reabilitação psicossocial, engloba todos os profissionais e todos os atores do processo saúde-doença, 
${ }^{4}$ Utiliza-se a convenção de se denominar at (acompanhante terapêutico) para o profissional que exerce a prática. ou seja, os usuários (portadores e familiares) e a comunidade inteira (Saraceno, 1999). O modelo de referência da reabilitação é o poder de contratualidade, resgatado pelo cidadão em meio ao cenário de seu habitat, cenário do mercado e o cenário do trabalho. A contratualidade inclui o poder de se realizarem trocas afetivas, sociais e econômicas (Pitta, 2001).

Nos dispositivos extra-hospitalares, então, incluem-se as práticas de atendimento psicossocial que não se limitam às paredes institucionais.

Destaca-se aqui a origem da clínica do AT, ideologicamente influenciada pela movimentação política das reformas psiquiátricas pela tentativa de supressão dos manicômios na Europa Ocidental, nos anos 60. Na América do Sul, na década de 1970, a Argentina utilizou o atendimento da abordagem múltipla em uma clínica de atendimento a pacientes psicóticos e dependentes químicos. Por meio dessa abordagem, os pacientes e familiares recebiam atendimento de equipe multiprofissional, aparecendo, pela primeira vez e de uma maneira sistematizada, a prática do $\mathrm{AT}$, sendo o profissional acompanhante terapêutico um dos membros dessa equipe (Mauer, Resnizky, 1987).

O AT é um tipo de atendimento clínico que se caracteriza pela prática de saídas pela cidade, ou estar ao lado da pessoa em dificuldades psicossociais com a intenção de se montar um guia terapêutico que possa articulá-la novamente na circulação social, por meio de ações sustentadas numa relação de vizinhança do acompanhante com o sujeito e suas limitações, dentro do seu contexto histórico. O profissional, acompanhante terapêutico (at) ${ }^{4}$, não está atrelado a uma profissão em particular, mas necessita de qualificação específica para exercício dessa prática profissionalmente (Pitiá, Santos, 2005; Berger, 1997).

No Brasil, no final dos anos 70, essa prática seguiu dois trajetos: Porto Alegre, depois Rio de Janeiro, com a figura dos auxiliares psiquiátricos, e São Paulo, com os chamados "amigos qualificados", sob influência direta da Argentina. Esses antigos "auxiliares psiquiátricos", na clínica de Vila Pinheiros, na cidade do Rio de Janeiro, por volta do início da década de 1980, recebiam curso de 24 meses dentro da própria instituição, sendo constituída de estudantes das várias áreas da saúde (Reis Neto, 1995).

Com o objetivo terapêutico de inclusão social, congruência com os princípios da Reforma Psiquiátrica e reabilitação psicossocial, o AT é uma atividade clínica, em movimento, que procura desinstalar o indivíduo de sua situação de dificuldade para poder recriar algo de novo na sua condição. O projeto terapêutico não é aleatório, apesar da vivência de cenas inesperadas. Há a importância de um guia, de uma proposta terapêutica, que procura articular a pessoa em seu espaço social. É um processo de reinvenção e se faz presente a partir da própria condição do sujeito(s) acompanhado(s) (Pitiá, Santos, 2005). O AT constitui-se em uma atividade clínica porque, pela relação terapêutica entre o profissional (acompanhante terapêutico) e o acompanhado, a ação ocorre em sintonia com a execução do projeto terapêutico que favorece o processo de reabilitação psicossocial do sujeito atendido.

Considerando a produção de um novo projeto de atendimento, voltado para os princípios antissegregacionistas, o dispositivo clínico do AT tem a marca da interdisciplinaridade, podendo se associar como uma estratégia a mais em serviços tais como os Centros de Atenção Psicossocial (os CAPS) e os Ambulatórios de Saúde Mental, e, mesmo, os serviços de internação (Pitiá, Santos, 2005) - caracterizados pela prática multiprofissional e articulados a saberes de Enfermagem, Medicina, Psicologia, Serviço Social e Terapia Ocupacional voltados ao atendimento e solução dos problemas pessoais, grupais e sociais, determinantes e determinados por alterações do comportamento e da afetividade. 
A interdisciplinaridade é marca característica da ação terapêutica contemporânea, promotora do processo de inclusão social de sujeitos em dificuldades psicossociais. Nesse eixo, giram os diversos saberes, encontrando-se campo fértil para o processo terapêutico em saúde mental (Sampaio, Santos, 2001; Maia, Pirim, 1997).

O AT pode ser reconhecido e incluído como mais um dispositivo de atendimento nos serviços de saúde, em vista de que trabalha com projetos terapêuticos que favorecem o resgate do poder de contratualidade social, sob os princípios psicossociais de reabilitação. Pelliccioli e Bernardes (2003) confirmam essa idéia, entendendo a prática do AT como dispositivo complementar que contribui para a efetivação dos princípios da Reforma Psiquiátrica, em que o espaço público é compreendido como alargamento do campo possível de tratamento para pacientes portadores de transtorno mental e sofrimento psíquico.

Mauer e Resnizky (1987) sistematizaram pela primeira vez a prática do AT, referindo-se à importância interdisciplinar e sua inclusão na equipe de saúde mental. Posteriormente, Maia e Pirim (1997) evidenciaram o aspecto da singularidade que se busca no processo de reinserção psicossocial - o que é considerado no AT. Estudos, como os de Barreto (2005) e Pitiá e Santos (2005), corroboram a aplicabilidade e a expansão da prática do AT, por meio de pesquisas que trazem novas perspectivas para o tratamento de portadores de sofrimento psíquico, consoante com os princípios do modo de atenção psicossocial.

Os atendimentos em AT podem ocorrer uma, duas ou mais vezes por semana e duram entre uma a três horas. Nesse espaço de tempo, o acompanhante terapêutico (at) e a pessoa acompanhada realizam atividades que promovam o alcance dos objetivos terapêuticos, para restabelecimento da conexão da pessoa com o mundo circundante, demandada pelas suas necessidades de inclusão no espaço social. Assim, ir ao supermercado, ir ao banco, ir à padaria, à lanchonete, procurar um emprego, e assim por diante, são exemplos de ações cotidianas realizáveis neste projeto que mobiliza o resgate de ações que foram bloqueadas pela dificuldade instalada na pessoa, para as quais ela necessita de ajuda. O significado dessas ações é trabalhado pelo profissional at, durante os atendimentos, o que exige um nível de maturidade profissional para apreender a complexidade da realização de atividades, aparentemente simples, em meio à proteção do setting terapêutico, que se constitui na própria relação entre o at e o acompanhado.

Profissionais que tenham a intenção de trabalhar com a prática AT, necessitam de formação e estudos que os preparem para esta atividade clínica. No âmbito dessa formação específica (a função de acompanhantes terapêuticos), os profissionais das diversas categorias devem ser levados a refletirem e agirem, na prática, em consonância com os princípios transformadores da forma clássica dos atendimentos psiquiátricos. Além disso, as diversas abordagens teóricas que procuram dar conta das análises dos atendimentos devem considerar os vários campos de ação terapêutica, seja o biopsíquico, o político, o social e o cultural do portador de transtorno mental em acompanhamento.

O desafio é a consolidação de uma rede de atendimentos que possibilite perspectivas de vida aos portadores de sofrimento psíquico, levando-se em conta o sujeito aí implicado e o contexto em que se encontra imerso (Palombini, 2004).

As práticas em saúde mental, com destaque para o AT, contam também com o desafio da interdisciplinaridade, ou seja, é necessário um esfumaçamento dos limites e atribuições específicas de cada disciplina ou profissão, o que diversifica e amplia os espaços de atuação de cada profissional, criando regiões novas no saber e o intercruzamento dos diversos conteúdos em saúde.

\section{Considerações finais}

Os atuais dispositivos de atendimento na rede pública de saúde mental facilitam o engendramento das ações dos diversos profissionais voltados para a singularidade como princípio de uma clínica antimanicomial. Quando a intervenção clínica for pertinente, a concepção do singular deverá ser tomada em consideração e, sua interpelação, levará o sujeito atendido a sustentá-la com seu estilo pessoal. 
Esta proposta é contrária à visão de uma simples adaptação do indivíduo à sociedade. É necessário que uma intervenção terapêutica o convide a sustentar sua diferença, sem precisar excluir-se do social. Daí, a consideração de que o trabalho clínico no AT deva conduzir o tratamento de forma tal que o sujeito siga o caminho que lhe seja próprio - mantendo-o, ao mesmo tempo, dentro dos limites cabíveis da cultura.

A rede de atendimentos - quais sejam os dispositivos hospitalares, quando for o caso, e os comunitários (ambulatórios, CAPS, hospitais-dia, oficinas etc..), voltados para a concepção de uma clínica antimanicomial -, exige transformações sociais para além de questionamentos sobre a exclusão. Os princípios antimanicomiais, por si só, não se constituem em processos de inclusão do sujeito que apresenta dificuldades psicossociais. É necessária a mudança de atitude dos profissionais da saúde e sua efetivação nas práticas.

A clínica do Acompanhamento Terapêutico, inserida no contexto das práticas do modo contemporâneo de atenção psicossocial, por sua característica interdisciplinar e antissegregacionista, exige formação específica do profissional na direção de mudanças de concepções, de atitudes e de comportamentos e mentalidade para melhor eficácia nos atendimentos em saúde mental.

É preciso enfrentar o desafio da participação colaborando na construção de uma nova rede de atendimento em saúde, contribuindo com estudos associados às práticas clínicas comunitárias, nas quais o AT, interdisciplinarmente, vem se consolidando em consonância com as transformações das políticas públicas em saúde, baseadas na atenção psicossocial e seus vários dispositivos de intervenção terapêutica.

\section{Colaboradores}

Ana Celeste de Araújo Pitiá foi responsável pela elaboração do texto, prática e pesquisa no tema. À autora Antonia Regina Ferreira Furegato coube a orientação teórica e coelaboração do texto.

\section{Referências}

ALVES, D.S.N. Por um programa brasileiro de apoio à desospitalização. In: PITTA, A. (Org.). Reabilitação psicossocial no Brasil. 2.ed. São Paulo: Hucitec, 2001. p.27-30.

AMARANTE, P. Manicômio e loucura no final do século e do milênio. In: FERNANDES, M.I.(Org.). Fim de século: ainda manicômios? São Paulo: IPUSP, 1999. p. 47-56.

(Org.). Loucos pela vida: a trajetória da Reforma Psiquiátrica no Brasil. Rio de Janeiro: Panorama Ensp, 1995.

ARANHA E SILVA, A.L.; FONSECA, R.M.G.S. Processo de trabalho em saúde mental e o campo psicossocial. Rev. Latino-Am. Enferm., v.3, n.13, p.441-9, 2005.

BARRETO, K.D. Ética e técnica no acompanhamento terapêutico: andanças com Dom Quixote e Sancho Pança. 3.ed. São Paulo: Unimarco/Edições Sobornost, 2005.

BERGER, E. Acompanhamento terapêutico: invenções. In: EQUIPE DE ACOMPANHANTES TERAPÊUTICOS DE A CASA (Org.). Crise e cidade: acompanhamento terapêutico. São Paulo: Educ, 1997. p.71-82.

BRASIL. Ministério da Saúde. Secretaria Executiva. Secretaria de Atenção à Saúde. Legislação em saúde mental: 1990-2004. 5.ed. Brasília: Ministério da Saúde, 2004. 
BRONZ, A. et al. Acompanhamento Terapêutico: uma prática em construção. VENÂNCIO, A.T.; LEAL, E.M.; DELGADO, P.G. (Orgs.). In: CONGRESSO DE SAÚDE MENTAL DO ESTADO DO RIO DE JANEIRO, 1., 1997, Rio de Janeiro. Anais... Rio de Janeiro: Te Corá Editora, 1997. p.15-20.

COSTA-ROSA, A.; LUZIO, C.A.; YASUI, S. As Conferências Nacionais de Saúde Mental e as premissas do modo psicossocial. Saúde em Debate, v.25, n.58, p.12-25, 2001.

COSTA-ROSA, A. O modo psicossocial: um paradigma das práticas substitutivas ao modo asilar. In: AMARANTE, P. (Org.). Ensaios: subjetividades, saúde mental, sociedade. Rio de Janeiro: Fiocruz, 2000. p.141-68.

FOUCAULT, M. História da loucura. São Paulo: Perspectiva, 1993.

FUREGATO, A.R.F. A conduta humana e a trajetória do ser e do fazer da enfermagem. In: JORGE, M.S.B.; SILVA, W.V.; OLIVEIRA, F.B.O. (Orgs.). Saúde mental: da prática psiquiátrica asilar ao terceiro milênio. São Paulo: Lemos Editorial, 2000. p.93-116.

LOBOSQUE, A.M. Princípios para uma clínica antimanicomial e outros escritos. São Paulo: Hucitec, 1997.

MAIA, M.S.; PIRIM, M. Em busca de uma singularidade: uma proposta clínica no processo de reinserção psicossocial. VENÂNCIO, A.T.; LEAL, E.M.; DELGADO, P.G. (Orgs.). In: CONGRESSO DE SAÚDE MENTAL DO ESTADO DO RIO DE JANEIRO, 1., 1997, Rio de Janeiro. Anais... Rio de Janeiro: Te Corá, 1997. p. 5-14.

MAUER, S.; RESNIZKY, S. Acompanhamento terapêutico e pacientes psicóticos: manual introdutório a uma estratégia clínica. Trad. Waldemar Paulo Rosa. Campinas: Papirus, 1987.

ONOCO-CAMPOS, R.T.; FURTADO, J.P. Entre a saúde coletiva e a saúde mental: um instrumental metodológico para avaliação da rede de Centros de Atenção Psicossocial (CAPS) do Sistema Único de Saúde. Cad. Saude Publica, n.22, v.5, p.1053-62, 2006.

ORGANIZACIÓN PANAMERICANA DE LA SALUD - OPS. La enfermería contribuyendo a la salud mental en las Américas: asociándonos para el progreso. Geneva: Opas, 2003.

OSINAGA, V.L.M.; FUREGATO, A.R.F.; SANTOS, J.L.F. Concepts of mental health and care according to mentally - III patients and their relatives. Rev. Latino-Am. Enferm., v.3, n.13, p.332-8, 2005.

PALOMBINI, A.L. et al. Acompanhamento terapêutico na rede pública: a clínica em movimento. Porto Alegre: Editora da UFRGS, 2004.

PELLICCIOLI, E.C.; BERNARDES, A.G. O trabalhador da saúde mental na rede pública: o acompanhamento terapêutico na rede pública. In: SEMINÁRIO INTERNACIONAL DE EDUCAÇÃO INTERCULTURAL: GÊNERO E MOVIMENTOS SOCIAIS, 2. 2003, Florianópolis. Anais... Florianópolis, 2003. p.1-13.

PITIÁ, A.C.A. Acompanhamento Terapêutico sob o enfoque da psicoterapia corporal: uma clínica em construção. 2002. Tese (Doutorado - Doenças crônicas, repercussões psicológicas e intervenção psicoterapêutica) - Escola de Enfermagem de Ribeirão Preto, Universidade de São Paulo, Ribeirão Preto. 2002.

O enfermeiro e seu cotidiano: cenas de um manicômio. 1997. Dissertação

(Mestrado - Enfermagem psiquiátrica) - Escola de Enfermagem de Ribeirão Preto, Universidade de São Paulo, Ribeirão Preto. 1997.

PITIÁ, A.C.A.; SANTOS, M.A. Acompanhamento terapêutico: a construção de uma estratégia clínica. São Paulo: Vetor, 2005.

PITTA, A.M.F. O que é reabilitação psicossocial no Brasil, hoje? In: (Org.)

Reabilitação psicossocial no Brasil. 2.ed. São Paulo: Hucitec, 2001. p.19-26. 
REIS NETO, R.O. Acompanhante terapêutico: trajetória histórica de uma prática em saúde mental no Rio de Janeiro. 1995. Dissertação (Mestrado) - Departamento de Psicologia, Pontifícia Universidade Católica do Rio de Janeiro, Rio de Janeiro. 1995.

SARACENO, B. Libertando identidades: da reabilitação psicossocial à cidadania possível. Instituto Franco Basaglia. Belo Horizonte/Rio de Janeiro: Te Corá, 1999.

A concepção de reabilitação psicossocial como referencial para as intervenções terapêuticas em saúde mental. Rev. Ter. Ocup. Univ., v.9, n.1, p.26-31, 1998.

SAMPAIO, J.J.C.; SANTOS, A.W.G. A experiência do Centro de Atenção Psicossocial e o Movimento Brasileiro de Reforma Psiquiátrica. In: PITTA, A. (Org.). Reabilitação psicossocial no Brasil. São Paulo: Hucitec, 2001. p.127-34.

SOUZA, R.C. Produção de sentidos por profissionais de Saúde da Família acerca do doente mental e dos cuidados a ele dirigidos. 2004. Tese (Doutorado) - Escola de Enfermagem de Ribeirão Preto, Universidade de São Paulo, Ribeirão Preto. 2004.

STEFANELLI, M.C. Enfermagem psiquiátrica no século XXI. In: STEFANELLI, M.C.; FUKUDA, I.M.K.; ARANTES, E.C. (Orgs.). Enfermagem psiquiátrica em suas dimensões assistenciais. Barueri: Manole, 2008. p.3-15. (Série Enfermagem).

VIANNA, P.C.M.; BARROS, S. O papel das políticas sociais frente à reestruturação do modelo assistencial em saúde mental. Saúde em Debate, v.29, n.69, p.27-35, 2005.

WORLD HEALTH ORGANIZATION - WHO. Relatório Mundial de Saúde - Saúde mental: nova concepção, nova esperança. Lisboa: Climeps, 2002.

PITIÁ, A.C.A.; FUREGATO, A.R.F. El Acompañamiento Terapéutico (AT): dispositivo de atención psico-social en salud mental. Interface - Comunic., Saude, Educ., v.13, n.30, p.67-77, jul./set. 2009.

Este artículo aborda la práctica clínica del Acompañamiento Terapéutico (AT) incorporado al modo de atención psico-social en salud mental y sus otros dispositivos. Se trata de discutir el proceso de rehabilitación psico-social y este tipo de acción terapéutica en la salud mental a partir de la clínica de AT. Para ello se realizó una reflexión teórica considerando los paradigmas de los modos de asilar y psico-social de atención que hacen referencia a las prácticas de intervención terapéutica en salud mental. Las reflexiones conducen a la importancia de la consolidación de una red de atención que posibilite perspectiva de vida a los portadores de sufrimiento psíquico como un desafío a enfrentar que considere prioritariamente el sujeto implicado y su contexto de inmersión social.

Palabras clave: Acompañamiento terapéutico. Rehabilitación psico-social. Enfermería psiquiátrica. Salud mental. 
